EPJ Web of Conferences 106, 04002 (2016)

DOI: $10.1051 /$ epjconf/201610604002

(C) Owned by the authors, published by EDP Sciences, 2016

\title{
Toward a New Evaluation of Neutron Standards
}

\author{
A.D. Carlson 1, a, V.G. Pronyaev ${ }^{2}$, R. Capote ${ }^{3}$, G.M. Hale ${ }^{4}$, F.-J. Hambsch ${ }^{5}$, T. Kawano ${ }^{4}$, S. Kunieda ${ }^{6}$, \\ W. Mannhart ${ }^{7}$, R.O. Nelson ${ }^{4}$, D. Neudecker ${ }^{4}$, P. Schillebeeckx ${ }^{5}$, S. Simakov ${ }^{3}$, D.L. Smith ${ }^{8}$, P. Talou ${ }^{4}$, \\ X. Tao ${ }^{9}$, A. Wallner ${ }^{10}$, and W. Wang ${ }^{9}$ \\ ${ }^{1}$ NIST, 100 Bureau Drive Stop 8463, Gaithersburg, MD 20899-8463, USA \\ ${ }^{2}$ IPPE, Bondarenko Sq. 1, 249033 Obninsk, Kaluga Region, Russia \\ ${ }^{3}$ International Atomic Energy Agency, NAPC-Nuclear Data Section, Vienna, Austria \\ ${ }^{4}$ Los Alamos National Laboratory, Los Alamos, NM 87545, USA \\ ${ }^{5}$ EC-JRC-IRMM, Retieseweg 111, 2440 Geel, Belgium \\ ${ }^{6}$ Japan Atomic Energy Agency, Nuclear Data Center, 2-4 Shirane Shirakata Tokai-mura, Naka-gun, \\ Ibaraki 319-1195, Japan \\ ${ }^{7}$ Physikalisch-Technische Bundesanstalt, Org. 6.4, Bundesallee 100, 38116 Braunschweig, Germany \\ ${ }^{8}$ Argonne National Laboratory, 9700 South Cass Avenue, Argonne, IL 60439, USA \\ ${ }^{9}$ China Institute of Atomic Energy, China Nuclear Data Center (CNDC), P.O. Box 275(41) Beijing \\ 102413, China \\ ${ }^{10}$ The Australian National University, Research School of Physics and Engineering, Building 57, \\ Nuclear Physics' ACT 0200, Canberra, Australia
}

\begin{abstract}
Measurements related to neutron cross section standards and certain prompt neutron fission spectra are being evaluated. In addition to the standard cross sections, investigations of reference data that are not as well known as the standards are being considered. Procedures and codes for performing this work are discussed. A number of libraries will use the results of this standards evaluation for new versions of their libraries. Most of these data have applications in neutron dosimetry.
\end{abstract}

\section{Introduction}

An international effort is now underway to provide improved evaluations of the neutron standards. These will be the standards in new versions of several nuclear data libraries, including ENDF/B and JEFF. This effort has been supported by the Cross Section Evaluation Working Group from the United States, the Working Party on International Evaluation Cooperation of the Nuclear Energy Agency Nuclear Science Committee and the International Atomic Energy Agency (IAEA). An IAEA Data Development Project has provided essential support and a mechanism for allowing new experimental data and improvements in evaluation procedures to be incorporated into new evaluations of the standards. This international

\footnotetext{
${ }^{\text {a }}$ Corresponding author: carlson@nist.gov
}

This is an Open Access article distributed under the terms of the Creative Commons Attribution License 2.0, which permits unrestricted use, distribution, and reproduction in any medium, provided the original work is properly cited. 
effort provided the standards for the previous evaluation and is now preparing to produce the standards, within the next few years, for the next evaluation of the standards. Information on the development, energy ranges, uncertainties and availability of the standards can be found in [1].

\section{The Evaluation Process}

The standards evaluation includes work on each of the following: the neutron cross section standards; the ${ }^{252} \mathrm{Cf}$ spontaneous prompt fission neutron spectrum; the ${ }^{235} \mathrm{U}$ thermal neutron-induced prompt fission neutron spectrum; reference gamma-ray production cross sections; the low energy gold capture cross section.

\subsection{Neutron Cross Section Standards}

The standard cross sections are for the following reactions: $\mathrm{H}(\mathrm{n}, \mathrm{n}),{ }^{3} \mathrm{He}(\mathrm{n}, \mathrm{p}),{ }^{6} \mathrm{Li}(\mathrm{n}, \mathrm{t}),{ }^{10} \mathrm{~B}\left(\mathrm{n}, \alpha_{1} \gamma\right)$, ${ }^{10} \mathrm{~B}(\mathrm{n}, \alpha), \mathrm{C}(\mathrm{n}, \mathrm{n}), \mathrm{Au}(\mathrm{n}, \gamma),{ }^{235} \mathrm{U}(\mathrm{n}, \mathrm{f})$ and ${ }^{238} \mathrm{U}(\mathrm{n}, \mathrm{f})$. Also included in the evaluation process are the ${ }^{238} \mathrm{U}(\mathrm{n}, \gamma)$ and ${ }^{239} \mathrm{Pu}(\mathrm{n}, \mathrm{f})$ cross sections. Those data were included since there are many ratio measurements of those cross sections with the standards and absolute data are available for them. Then the evaluation process will, in addition to the standard cross sections, produce evaluations for the ${ }^{238} \mathrm{U}(\mathrm{n}, \gamma)$ and ${ }^{239} \mathrm{Pu}(\mathrm{n}, \mathrm{f})$ cross sections that are important for dosimetry and reactor applications.

\subsubsection{Recent Measurements}

Only new or continuing work will be included here. For information on the older work, see [2]. Significant work is underway or recently completed for the following measurements:

Daub et al. [3] made measurements of the hydrogen total cross section where very few measurements of that cross section have been made - at low neutron energies. The data were measured at the University of Kentucky Van de Graaff facility from $150 \mathrm{keV}$ to $800 \mathrm{keV}$. The results are systematically slightly larger than the evaluated values but generally within their uncertainties of 1.1 to $2 \%$ (note that all uncertainties in this paper are one standard deviation uncertainties). Additional work [4] at the Ohio University Accelerator Laboratory on the hydrogen standard now emphasizes the small angles in the center of mass system (CMS) at about 10 and $14 \mathrm{MeV}$ where very few data are available.

Accurate absolute measurements of the ${ }^{6} \mathrm{Li}(\mathrm{n}, \mathrm{t})$ cross section at $4 \mathrm{meV}$ by Yue et al. [5] at NIST are completed. The measurements are very consistent with the results of the most recent standards evaluation. Hambsch is now making measurements at the JRC-IRMM GELINA facility of the ${ }^{6} \operatorname{Li}(\mathrm{n}, \mathrm{t})$ cross section relative to the ${ }^{235} \mathrm{U}(\mathrm{n}, \mathrm{f})$ standard from a few $\mathrm{keV}$ to $3 \mathrm{MeV}$. Also at JRC-IRMM, but at the Van de Graaff facility, Giorginis and Bencardino have made measurements and are now analyzing ${ }^{6} \mathrm{Li}(\mathrm{n}, \mathrm{t})$ cross section data relative to the ${ }^{238} \mathrm{U}(\mathrm{n}, \mathrm{f})$ standard for the 1.8 to $2.1 \mathrm{MeV}$ region.

At JRC-IRMM, using GELINA, Hambsch is making branching ratio, angular distribution and cross section measurements for the ${ }^{10} \mathrm{~B}(\mathrm{n}, \alpha)$ reaction. This work will provide data up to about $3 \mathrm{MeV}$. See [6] for information on the ${ }^{6} \mathrm{Li}(\mathrm{n}, \mathrm{t})$ and ${ }^{10} \mathrm{~B}(\mathrm{n}, \alpha)$ work at JRC-IRMM. Measurements of the ${ }^{10} \mathrm{~B}\left(\mathrm{n}, \alpha_{1} \gamma\right)$, ${ }^{10} \mathrm{~B}\left(\mathrm{n}, \alpha_{0}\right),{ }^{10} \mathrm{~B}(\mathrm{n}, \mathrm{p})$ and ${ }^{10} \mathrm{~B}(\mathrm{n}, \mathrm{t})$ cross sections have been made at the WNR facility at LANL relative to the ${ }^{238} \mathrm{U}(\mathrm{n}, \mathrm{f})$ standard [7]. Analysis of these data is still ongoing.

Carbon transmission measurements have been made by Gritzay et al. [8]. The results were shown to agree with the standards evaluation and are not dependent on the sample thickness. A motivation for this work was to determine if a strong resonance predicted by Canton, et al. [9] is present in the 130-160 keV energy region. No evidence for a resonance was found. There are also measurements of the carbon total cross section by Anh, et al. [10] made with reactor filtered beams at 54 and $148 \mathrm{keV}$ that agree with the standards evaluation. Daub et al. [3] also made measurements of the carbon total cross section. They agree with the standard within uncertainties but are lower than it. 


\section{$15^{\text {th }}$ ISRD}

The work on gold capture by Wallner et al. [11] who made a ${ }^{238} \mathrm{U}(\mathrm{n}, \gamma) / \mathrm{Au}(\mathrm{n}, \gamma)$ cross section ratio measurement at $430 \mathrm{keV}$ is being published. The experiment used accelerator mass spectrometry to measure the ${ }^{239} \mathrm{Pu}$ resulting from the decay of ${ }^{239} \mathrm{U}$. This new independent method will not have some unknown systematic uncertainties that may be present in other measurements. The cross section ratio obtained agrees with the standards evaluation. ${ }^{238} \mathrm{U}(\mathrm{n}, \gamma)$ cross section measurements have been made recently at the GELINA and $n_{-}$TOF facilities using the same sample. At GELINA, Lampoudis et al. [12] made measurements using a $\mathrm{C}_{6} \mathrm{D}_{6}$ detector. At $\mathrm{n}_{-} \mathrm{TOF}$, the measurements were made with a different $\mathrm{C}_{6} \mathrm{D}_{6}$ detector by Mingrone et al. [13] and with a $\mathrm{BaF}_{2}$ detector by Wright et al. [14]. They anticipate average values will be available from $20 \mathrm{keV}$ to $1 \mathrm{MeV}$. The measurements by Ullmann et al . of the ${ }^{238} \mathrm{U}(\mathrm{n}, \gamma)$ cross section at the WNR and LANSCE facilities at LANL have been published [15]. They agree with the standards evaluation over their entire energy range $-10 \mathrm{eV}$ to $100 \mathrm{keV}$.

Measurements are now being published [16] for the ${ }^{238} \mathrm{U}(\mathrm{n}, \mathrm{f}) /{ }^{235} \mathrm{U}(\mathrm{n}, \mathrm{f})$ cross section ratio by Calviani et al. using a fission chamber and by Audouin et al. using a parallel plate avalanche counter. The Calviani data are about 3\% higher than the Audouin data. The standards evaluation falls between these two data sets. Considering the uncertainties, the data sets are in good agreement with the standards evaluation. An experiment has been designed at the University of Kentucky and preliminary measurements have been made of the ${ }^{238} \mathrm{U}(\mathrm{n}, \mathrm{f})$ cross section by Miller and Kovash [17]. The data are being measured relative to hydrogen scattering for neutron energies above $100 \mathrm{MeV}$ at WNR.

Measurements have been made at LANL of the ${ }^{239} \mathrm{Pu}(\mathrm{n}, \mathrm{f})$ cross section by Tovesson and Hill [18]. The data are relative to the ${ }^{235} \mathrm{U}(\mathrm{n}, \mathrm{f})$ cross section. They agree well with the standards evaluation up to about $10 \mathrm{MeV}$. Above that the measurements are somewhat lower than the standards evaluation.

Very accurate fission cross section ratio measurements that include ${ }^{239} \mathrm{Pu}(\mathrm{n}, \mathrm{f})$ data are being measured at the LANL WNR facility. The data are being obtained with a Time Projection Chamber in a collaboration headed by LANL and LLNL. Some data analysis has been done.

\subsubsection{Evaluation of the Standard Cross Sections}

The evaluation process for the new evaluation [19] is expected to be similar to that used for the previous standards evaluation. For more information and references to the work in this subsubsection also see [19]. The hydrogen evaluation is expected to be extended to $200 \mathrm{MeV}$.

For details on the previous evaluation process see [1]. At this time it is not clear how many Rmatrix evaluations will be used. The EDA R-matrix code of Hale is expected to be used. There is also an R-matrix code AMUR by Kunieda that may be used in the evaluation effort if it is updated to include polarization data. The R-matrix code FDRR by Tao and Wang may be used if several actions are satisfied including use of systematic and statistical uncertainties, and production of the covariance matrix of evaluated cross sections as output.

The GMAP code will be used for the generalized least-squares simultaneous evaluation. This code provides the combining procedure by using input from the separate R-matrix analyses and a thermal constants evaluation in addition to the direct data sets normally used. The R-matrix input and thermal constants data will be treated like the additions of other data sets. The covariances and cross sections will be directly obtained from this evaluation procedure as is required by the dosimetry community.

\subsection{Prompt Neutron Fission Spectra Standards}

The ${ }^{252} \mathrm{Cf}$ spontaneous prompt fission neutron spectrum $\left({ }^{252} \mathrm{Cf}\right.$ ( $\mathrm{sf}$ ) PFNS) is a standard that is used in measurements of neutron fluence. The PFNS of ${ }^{235} U$ for thermal neutrons $\left({ }^{235} U\left(n_{t h}, f\right)\right)$, though it is not a standard, is being treated as reference data since it is important for nuclear energy applications. It is also used as a spectrum for dosimetry testing of cross sections. 


\subsection{1 ${ }^{252} \mathrm{Cf}$ (sf) PFNS}

There have been no recent measurements of the ${ }^{252} \mathrm{Cf}$ (sf) PFNS. The most recent evaluation [20] of that spectrum is based on all suitable experimental data available in 1987. There are problems with more recent measurements. Due to the lack of suitable data sets and inconsistencies in the new data sets, it was felt that a new evaluation is not justified.

\subsection{2 ${ }^{235} \mathrm{U}\left(\mathrm{n}_{\mathrm{th}}, \mathrm{f}\right)$ PFNS}

There are two relatively new measurements of the PFNS of ${ }^{235} \mathrm{U}\left(\mathrm{n}_{\mathrm{th}}, \mathrm{f}\right)$. These efforts were motivated by the concern that calculations of this spectrum do not agree in detail with measurements, particularly at high and at low outgoing neutron energies.

The first of these consists of the measurements by Kornilov et al. [21] in a JRC-IRMM and IKI collaboration at the Budapest Research Reactor. An ionization chamber containing a ${ }^{235} \mathrm{U}$ sample, as well as a ${ }^{252} \mathrm{Cf}$ reference sample outside of the neutron beam was used in the experiment. The data obtained disagree in some respects with PFNS data for ${ }^{235} \mathrm{U}\left(\mathrm{n}_{\mathrm{th}}, \mathrm{f}\right)$ from different evaluated data libraries. However, the data agree well with most experimental results. The results show that the spectrum is softer, having a higher yield in the energy range below $1 \mathrm{MeV}$. It also has a larger yield above about $9 \mathrm{MeV}$ but the uncertainties are quite large in that energy region.

Measurements were also made of the ${ }^{235} \mathrm{U}\left(\mathrm{n}_{\mathrm{th}}, \mathrm{f}\right)$ PFNS for thermal neutrons relative to the ${ }^{252} \mathrm{Cf}$ (sf) PFNS at the Gatchina research reactor [22]. The measurements of the prompt neutron spectra were performed at 11 fixed angles between the neutron and light fragment direction in the range from $0^{\circ}$ to $180^{\circ}$ in $18^{\circ}$ intervals. The comparison of the data with experimental results obtained by other groups, including the Kornilov et al. results, shows good agreement (within experimental errors) among all experimental data in the $1.5-8 \mathrm{MeV}$ energy range. However, there is some discrepancy outside that energy region. Generally, the results obtained are consistent with the ENDF/B-VII.0 PFNS within the limits of the uncertainty. Again, the spectrum at low energies is softer than the evaluation however the agreement at high energies is good. The energy and angular correlated differential data from this experiment can be used for improving models under consideration for calculation of prompt fission neutron emission.

In addition to this recent work, only three TOF experiments of the PFNS of ${ }^{235} U\left(n_{t h}, f\right)$ PFNS have been performed since 1975. The level of documentation of the older experiments is poor which makes it difficult to generate quality covariance matrices for the data. Documented data have been obtained on the work by Starostov et al. [23]. For this measurement series, uncertainty information and an experimental set-up description are available but to some extent incomplete.

The spectra for neutrons emitted at energies $<10 \mathrm{MeV}$ are in contradiction to spectrum-averaged cross section data.

The quality of the experimental database for ${ }^{252} \mathrm{Cf}$ (sf) PFNS is much better than that for ${ }^{235} \mathrm{U}\left(\mathrm{n}_{\mathrm{th}}, \mathrm{f}\right)$ PFNS. For both neutron spectra no recent TOF experiments have been done since 1991, except for the work described here.

\subsubsection{Evaluations and Calculations of Spectra}

A combined non-model evaluation of the prompt fission neutron spectra for ${ }^{252} \mathrm{Cf}(\mathrm{s}, \mathrm{f})$ and ${ }^{235} \mathrm{U}\left(\mathrm{n}_{\mathrm{th}}, \mathrm{f}\right)$, using the GMA code is now underway [19]. There is also a separate Bayesian evaluation of the ${ }^{235} \mathrm{U}\left(\mathrm{n}_{\mathrm{th}}, \mathrm{f}\right)$ PFNS by Mannhart [19]. These two projects are ongoing. A combined preliminary fit to the ${ }^{235} \mathrm{U}\left(\mathrm{n}_{\mathrm{th}}, \mathrm{f}\right)$ PFNS as a ratio to a Maxwellian spectrum is compared to the ENDF/B-VII.0, Mannhart and Chen [24] results in Fig. 1. 


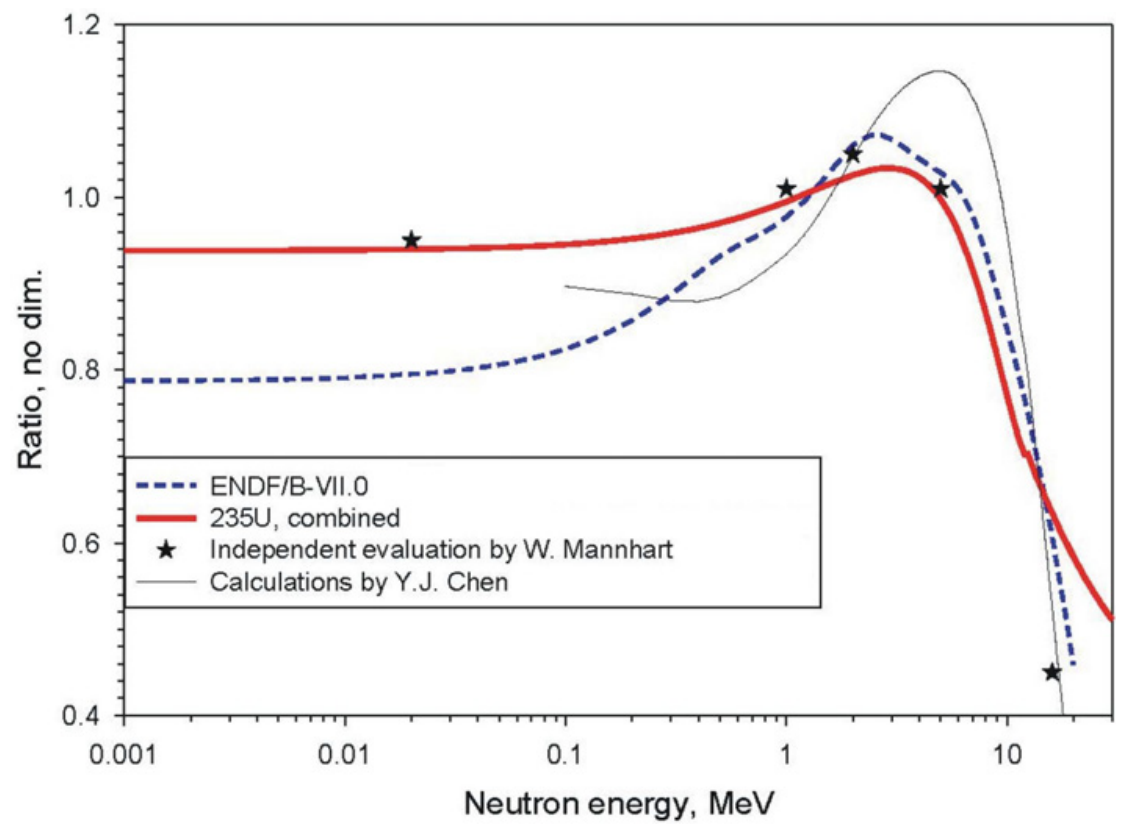

Figure 1. Comparison of a preliminary combined evaluation of the ${ }^{235} \mathrm{U}\left(\mathrm{n}_{\mathrm{th}}, \mathrm{f}\right)$, PFNS with other results.

A Unified Monte Carlo approach is being developed to perform model evaluations based on estimation of the model uncertainties used as a prior combined with a Bayesian least-squares fit of the experimental data. An attempt of this approach devoted to the uncertainty quantification of PFNS was developed by Rising et al. [25]. Traditionally, evaluations of PFNS rely on some version of the Los Alamos Madland-Nix model [26]. Recently, Monte Carlo calculations following the evaporation of excited fission fragments by successive evaporations of neutrons and $\gamma$ rays were performed by Talou [27] et al., Vogt [28] et al. and Litaize [29] et al. Within this new approach, physical quantities beyond the average PFNS and neutron multiplicity can be studied. When provided with accurate primary fission fragment yields, this approach has been shown to compute the average neutron multiplicity and spectrum on a par with existing evaluations, while also predicting exclusive and correlated data impossible to infer from simpler theoretical approaches. Full Monte Carlo Hauser-Feshbach calculations have been implemented by Kawano et al. [30], and they are being used to predict prompt gamma-ray multiplicity and spectrum. Those event-by-event models are more realistic than the Los Alamos model, but still face important challenges. They make several important assumptions regarding the mechanisms of emission, e.g., neutron emission from fully accelerated fragments only, and are sensitive to initial input parameters, e.g., fission fragment yields and nuclear structure, for which experimental data are lacking. Quantifying realistic uncertainties associated with this type of calculations also remains to be addressed.

In a parallel work, uncertainties associated with current evaluated PFNS that use the Los Alamos model calculations have been quantified for ${ }^{239} \mathrm{Pu}\left(\mathrm{n}_{\mathrm{th}}, \mathrm{f}\right)$ PFNS by Talou et al. [31]. A new evaluation effort is in progress at LANL, paying particular attention to experimental uncertainties and correlations. In addition, a more consistent PFNS evaluation procedure applicable to many isotopes is being developed in collaboration with the IAEA. These efforts are being extended to other PFNS of interest, such as ${ }^{235} \mathrm{U}\left(\mathrm{n}_{\mathrm{th}}, \mathrm{f}\right)$ and ${ }^{252} \mathrm{Cf}(\mathrm{sf})$. The evaluation of the spectrum for ${ }^{235} \mathrm{U}\left(\mathrm{n}_{\mathrm{th}}, \mathrm{f}\right)$ PFNS and the calculational efforts in this project will contribute to the CRP on evaluation of PFNS for a wide range of incident neutron energies and for a number of nuclides. 


\subsection{Gamma-ray Production Cross Sections}

Measurements of neutron-induced $\gamma$-ray production cross sections are most easily performed using a reference cross section in which a discrete $\gamma$-ray is detected. The need exists for accurate reference cross sections for such measurements. The preliminary conclusion is that the best candidates are ${ }^{\text {nat }} \mathrm{LiF}$ and ${ }^{\text {nat }} \mathrm{Ti}$. For ${ }^{\text {nat }} \mathrm{LiF}$, the ${ }^{7} \mathrm{Li}(\mathrm{n}, \mathrm{n}$ ') reaction has a yield that is isotropic; has little structure in the energy region 1 to $4 \mathrm{MeV}$; and the cross section is reasonably large. For ${ }^{\text {nat }} \mathrm{Ti}(\mathrm{n}, \mathrm{n}$ '), large yields of the $984 \mathrm{keV}$ $\gamma$ from ${ }^{48} \mathrm{Ti}\left(\mathrm{n}, \mathrm{n}^{\prime}\right)$ are very suitable for such use. New gamma-production cross section data for ${ }^{\text {nat }} \mathrm{Li}$ and ${ }^{\text {nat }} \mathrm{Ti}$ with GEANIE at LANSCE by Nelson et al. and at JRC-IRMM by Plompen et al. are in progress or planned. For current experiments, it is estimated that absolute cross sections can be obtained with uncertainties in the 2 to $3 \%$ range. Correlations exist due to the use of HPGe detectors and fission flux monitors in current experiments. Theoretical and GMA evaluation analyses performed on the basis of available experimental data have shown that the $984 \mathrm{keV} \gamma$-ray production cross section from the ${ }^{48} \mathrm{Ti}(\mathrm{n}, \mathrm{n}$ ') reaction can be estimated with an uncertainty of about $5 \%$ from threshold up to $20 \mathrm{MeV}$ [32]. However, the database for this evaluation is very limited.

\subsection{The Low Energy Gold Cross Section}

The Maxwellian averaged cross section for ${ }^{197} \mathrm{Au}(\mathrm{n}, \gamma)$ (MACS) is used in neutron capture crosssection measurements as a reference for reactions important for astrophysics, reactor and dosimetry applications. This reference cross section was obtained from an evaluation based on the results of measurements by Ratynski and Käppeler [33] of the ${ }^{197} \mathrm{Au}(\mathrm{n}, \gamma)$ cross section averaged over a Maxwellian-like experimentally simulated spectrum with temperature near $30 \mathrm{keV}$ as well as from measurements by Macklin [34]. The standards evaluation is approximately $5 \%$ to $7 \%$ above the Ratynski and Käppeler evaluation. The standards evaluation of the gold capture cross section makes use of a large amount of data other than gold capture cross section measurements. It included ratio measurements to other standards. In effect more than 400 cross section data sets had an impact to some degree on the standards evaluation results. The ${ }^{197} \mathrm{Au}(\mathrm{n}, \gamma)$ cross-section is a standard from $200 \mathrm{keV}$ to $2.5 \mathrm{MeV}$. Cross sections were obtained below $200 \mathrm{keV}$, from the standards evaluation process for that cross-section, however they are not recommended for use as a standard due to structure in the cross section there.

Because of this discrepancy new experiments and re-analyses have been done in an attempt to resolve the problem. Work has been done on the $n \_$TOF data and new capture measurements have been made at the University of Vienna, PTB and JRC-IRMM.

Lederer et al. [35] extended the energy range of the analysis of the n_TOF measurements of Massimi et al. [36]. The results, both below the standards energy region and in the standards energy region, generally agree with those obtained from the ${ }^{197} \mathrm{Au}(\mathrm{n}, \gamma)$ standards evaluation within the uncertainty of the measurements. The MACS from these data at $30 \mathrm{keV}$ is $2 \%$ smaller than the MACS obtained from the standards evaluation and $4.7 \%$ higher than the one obtained by Ratynski and Käppeler. The uncertainty of the n_TOF MACS at $30 \mathrm{keV}$ is $3.6 \%$, thus there is very good agreement with the standards evaluation and still fair agreement with Ratynski and Käppeler, who quote 1.5\% uncertainty for their result. All MACS values are compared for a temperature of $30 \mathrm{keV}$.

Measurements by Wallner et al. [11] of ${ }^{235} \mathrm{U}(\mathrm{n}, \gamma)$ and ${ }^{238} \mathrm{U}(\mathrm{n}, \gamma)$ cross sections relative to the ${ }^{197} \mathrm{Au}(\mathrm{n}, \gamma)$ cross section were made for a Maxwell-Boltzman simulated spectrum expected to be equivalent to that of Ratynski and Käppeler. The accelerator mass spectrometry method was used. The absolute ratio measurements were converted using the gold capture cross section. Doing simultaneous activation measurements using samples of ${ }^{197} \mathrm{Au},{ }^{235} \mathrm{U}$ and ${ }^{238} \mathrm{U}$, allowed absolute ratios of the ${ }^{235} \mathrm{U}(\mathrm{n}, \gamma)$, ${ }^{238} \mathrm{U}(\mathrm{n}, \gamma)$ and ${ }^{197} \mathrm{Au}(\mathrm{n}, \gamma)$ cross sections to be determined. This is an important independent measurement of the ratio of the ${ }^{238} \mathrm{U}(\mathrm{n}, \gamma)$ to ${ }^{197} \mathrm{Au}(\mathrm{n}, \gamma)$ cross sections. That ratio agrees with the standards evaluation. 


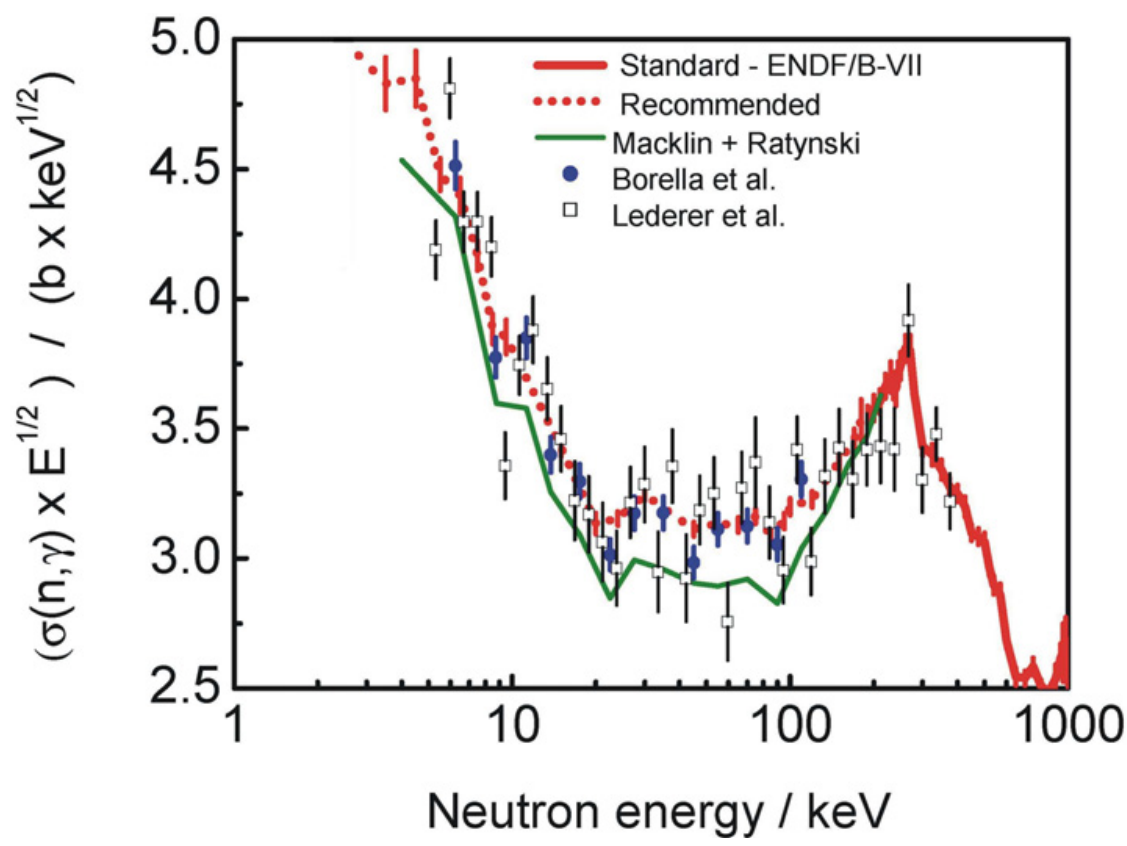

Figure 2. Comparsion of several ${ }^{197} \mathrm{Au}(\mathrm{n}, \gamma)$ cross section measurements and evaluations. The recommended data are the present work below the standards energy region.

New measurements of the ${ }^{197} \mathrm{Au}(\mathrm{n}, \gamma)$ cross section were made by Massimi et al. [37] at the GELINA facility. Large attention was paid to the measurements and analysis of the background, self-shielding and scattering corrections in this energy range. This led to a very small total measurement uncertainty of $1.5 \%$. The result agreed with the standards evaluation to within $2 \%$. Earlier work at the GELINA facility by Borella et al. [38] are also in good agreement with the standards evaluation.

Figure 2 shows a comparison of the ENDF/B-VII standard and the recommended results below the standards energy region with the Ratynski- Käppeler (and Macklin) evaluation and measurements by Borella and Lederer.

A spectrum averaged ${ }^{197} \mathrm{Au}(\mathrm{n}, \gamma)$ cross section measurement by Feinberg et al. [39] ] at JRC-IRMM is in good agreement with that calculated from the standards evaluation. It is about two standard deviations from the Ratynski and Käppeler value.

All these experiments agree with the standards evaluation.

Two measurements have been made of the the simulated Maxwellian spectrum used in the Ratynski and Käppeler measurements. Both experiments used neutrons from the ${ }^{7} \operatorname{Li}(\mathrm{p}, \mathrm{n})$ reaction for $\mathrm{E}_{\mathrm{p}}=1912 \mathrm{keV}$ (the same as that used by Ratynski and Käppeler). The spectrum measurements at PTB by Lederer et al. [40] are slightly softer, but have an effect of only $0.5 \%$ on the averaged Au cross section. A comparison with thick target yields calculated using the PINO [41] code and evaluated microscopic differential cross sections give good agreement with the results of this experiment. Independently, measurements of the neutron spectrum at JRC-IRMM by Feinberg et al. [39] showed good agreement with the findings of Ratynski and Käppeler and of Lederer et al. [40]. These experiments indicate the discrepancy is not a result of the neutron spectrum.

\section{$2.5 \mathrm{Bi}(\mathrm{n}, \mathrm{f})$}

The ${ }^{209} \operatorname{Bi}(n, f)$ reaction has been suggested as a reference cross section [42, 43]. Such data are useful for dosimetry and the development of accelerator-driven systems. This cross section may be a useful 
reference in the neutron energy region above $50 \mathrm{MeV}$ since the excitation function has a threshold of about $25 \mathrm{MeV}$ which eliminates the influence of low energy neutrons.

Relying on several measurements performed before 1997, an evaluation [43] of the reference cross sections for ${ }^{209} \mathrm{Bi}(\mathrm{n}, \mathrm{f})$ reaction from 21 to $1000 \mathrm{MeV}$ has been done with assigned uncertainties of $50 \%$ at neutron energies from 20 to $40 \mathrm{MeV}, 13-10 \%$ from 40 to $160 \mathrm{MeV}$ and about $30 \%$ above $169 \mathrm{MeV}$. More recent measurements have been performed up to $173 \mathrm{MeV}$ at Uppsala [44], up to $196 \mathrm{MeV}$ at Gatchina [45, 46], up to $150 \mathrm{MeV}$ in Louvain [47] and up $1000 \mathrm{MeV}$ in CERN [48]. These new experimental results make it reasonable to consider a new evaluation. Simakov, at the IAEA, will review the available experimental data and consider a new evaluation using GMA.

\section{Conclusions}

Standards are being evaluated for use in new data libraries. The cross sections and prompt neutron spectra being obtained in this work will generally be useful also to the dosimetry community.

\section{References}

[1] A.D. Carlson et al., Nucl. Data Sheets 100, 3215 (2009)

[2] A.D. Carlson, et al., J. ASTM Intl., 9, No. 4. doi: 10.1520/JAI104095 (2012)

[3] B.H. Daub et al., Phys. Rev. C87, 014005 (2013)

[4] N.V. Kornilov et al., Nucl. Data Sheets 119, 413 (2014)

[5] A. Yue et al., Bull. Am. Phys. Soc. DNP. CE. 7 (2010)

[6] R. Bevilacqua et al., Nucl. Data Sheets 119, 104 (2014)

[7] T.N. Massey et al., Nucl. Data Sheets 119, 107 (2014)

[8] O. Gritzay et al., Proceedings of the 4th International Conference "Current Problems in Nuclear Physics and Atomic Energy," Kiev Russia, http://www .kinr .kiev .ua/NPAE-Kyiv2012/ 439 (2008)

[9] L. Canton et al., Nuc. Phys. A790, 251 (2007)

[10] T.T. Anh et al., accepted for publication in Nucl. Data Sheets

[11] A. Wallner et al., Phys. Rev. Lett. 112, 192501 (2014)

[12] C. Lampoudis et al., Nucl. Data Sheets 119, 14 (2014)

[13] F. Mingrone et al., Nucl. Data Sheets 119, 18 (2014)

[14] T. Wright et al., Nucl. Data Sheets 119, 26 (2014)

[15] J.L. Ullmann et al. Phys. Rev. C89, 034603 (2014)

[16] M. Calviani, et al., private communication

[17] Z. Miller and M. Kovash, private communication

[18] F. Tovesson and T.S. Hill, Nucl. Sci. Eng. 165, 224 (2010)

[19] V.G. Pronyaev, A.D. Carlson and R. Capote Noy, IAEA Report INDC(NDS)-0641 (2013)

[20] W. Mannhart in http://www-nds.iaea.org/standards-cm-oct-2008/6. PDF

[21] N.V. Kornilov et al., Nucl. Sci. Eng. 165, 117 (2010)

[22] A.S. Vorobyev et al., "EFNUDAT Workshop Measurements and Models of Nuclear Reactions," EPJ Web of Conferences 8, 03004, Paris (2010)

[23] B.I. Starostov et al., Nejtronnaja Fizikaf 2, 285 (1983), EXFOR 40871, 40872, 40873 (1984)

[24] Y.J. Chen, IAEA Report INDC(NDS)-0608 (2014)

[25] M. Rising et al., Nucl. Sci. Eng. 175, 81 (2013)

[26] D.G. Madland and J.R. Nix, Nucl. Sci. Eng. 81, 213 (1982)

[27] P. Talou et al., Phys. Rev. C83, 064612 (2011)

[28] R. Vogt et al. Phys. Rev. C80, 044611 (2009)

[29] O. Litaize and O. Serot, Phys. Rev. C82, 054616 (2010) 


$$
15^{\text {th }} \text { ISRD }
$$

[30] T. Kawano et al., J. Nucl. Sci. Tech. 47, 462 (2010)

[31] P. Talou et al., Nucl. Sci. Eng. 166, 254 (2010)

[32] S.P. Simakov et al., Proc. of 13th Int. Conf. on Nuclear Reaction Mechanisms, Varenna, 11-15 June 2012, CERN-Proceedings-2012-002, 321 (2012)

[33] W. Ratynski and F. Käppeler, Phys. Rev. C 37, 595 (1988)

[34] R.L. Macklin, Nucl. Sci. Eng. 79, 265 (1981)

[35] C. Lederer et al., Phys. Rev. C83, 034608 (2011)

[36] C. Massimi et al., Phys. Rev. C81, 044616 (2010)

[37] C. Massimi, et al., IRMM, submitted to EPJA (2014)

[38] A. Borella et al., Nucl. Sci. Eng. 152, 1 (2006)

[39] G. Feinberg et al., Phys. Rev. C85, 055810 (2012)

[40] C. Lederer et al., Phys. Rev. C85, 055809 (2012)

[41] R. Reifarth et al., Nucl. Instr. and Meth. in Physics Research A 608, 139 (2009)

[42] I. Durán, private communication

[43] A.D. Carlson et al., INDC(NDS)-0368, IAEA (1997)

[44] A. N. Smirnov et al., Phys. Rev. C 70, 054603 (2004)

[45] O. Shcherbakov et al., J. Nucl. Sci. Technol. Suppl. 2, 230 (2002)

[46] A.B. Laptev et al., Conf. Fission. Prop. of Neutron-Rich Nuclei, Sanibel Island, World Scientific, p. 462 (2007)

[47] R. Nolte et al., J. Nucl. Sci. Technol. Suppl. 2, 311 (2002)

[48] D. Tarrio et al., Phys. Rev. C 83, 044620 (2011) 